X-641-68-60

PREPRINT

\title{
ELASTIC SCATTERING OF SLOW ELECTRONS \\ BY TWO-ELECTRON IONS
}

M. R. C. McDowell

February 1968

GODDARD SPACE FLIGHT CENTER

Greenbelt, Maryland 


\title{
PRECEDING PAGE BLANK NOT FILMED.
}

\author{
ELASTIC SCATTERING OF SLOW ELECTRONS \\ BY TWO-ELECTRON IONS
}

M. R. C. McDowell

\begin{abstract}
A one channel model, previously used for $\mathrm{He}$, is extended to allow calculation of elastic scattering by $\mathrm{H}^{-}$and $\mathrm{Li}^{+}$at energies up to three rydbergs. Nonseparable ground state wave functions are used, allowance being made for exchange, and dipole and quadrupole polarization potentials. Results for $\mathrm{Li}^{+}$are in good agreement with quantum defect method values. Calculated differential cross sections for elastic scattering by $\mathrm{H}^{-}$at energies of one half and one rydberg are presented. Deviations from Couloumb scattering are marked.
\end{abstract}




\title{
ELASTIC SCATTERING OF SLOW ELECTRONS
}

BY TWO-ELECTRON IONS

by

\author{
M. R. C. McDowell ${ }^{* \dagger}$ \\ NASA-Goddard Space Flight Center \\ Greenbelt, Maryland
}

\section{Introduction}

A considerable number of theoretical investigations on elastic scattering of slow electrons by He atoms ${ }^{1-4}$ have been reported recently. They are in substantial agreement with each other, and are consistent (in the sense of dispersion relations ${ }^{3}$ ) with experimental data. ${ }^{5,6}$ In view of advances in experimental technique which have allowed measurement ${ }^{7,8}$ of inelastic collision cross sections of slow electrons with $\mathrm{H}^{-}$(in good agreement with theory ${ }^{9}$ ) it is of interest to examine elastic scattering by two electron ions.

In this paper the model of Williamson and McDowell ${ }^{2}$ has been extended and applied to elastic scattering by $\mathrm{H}^{-}$and by $\mathrm{Li}^{+}$at energies up to $3 \mathrm{Ry}$. The theory is outlined in Section II, and the numerical methods employed are discussed in Section III. Phase shifts and scattered intensities are presented in Section IV,

\footnotetext{
${ }^{\star}$ National Academy of Sciences-National Research Council, Senior Post-doctoral Associate

tOn leave of absence from University of Durham, England 1967-8.
} 
and in the case of $\mathrm{Li}^{+}$compared with the result of a Hartree-Fock calculation ${ }^{10}$, and the quantum defect method.

\section{Theory}

We consider an electron scattered by a two electron system of charge $z$. The total three electron system is described by the Schrödinger equation,

$$
(\mathrm{H}-\mathrm{E}) \Psi(1,2,3)=0
$$

with Hamiltonian operator*

$$
H=\sum_{i=1}^{3}\left(-\frac{1}{2} \nabla_{i}^{2}-\frac{z}{r_{i}}\right)+\sum_{i>j} \frac{1}{r_{i j}} .
$$

In our model the total wave function (123) is represented by an ansatz,

$$
\Psi(1,2,3)=\sum_{1,2,3} \psi_{0}(1,2) \mathrm{F}(3) \mathrm{S}(123)
$$

where $\psi_{0}(1,2)$ is a wave function for the ground state of the target when electrons 1 and 2 are bound, $S(123)$ is a spin function, and $F(3)$ the unknown scattering function to be determined, and the sum is over cyclic permutations.

\footnotetext{
*All quantities will be given in atomic units, the unit of energy being the Rydberg, $13.565 \mathrm{eV}$.
} 
Taiking

$$
S(123)=\frac{1}{\sqrt{2}}\left(a_{1} \beta_{2}-\alpha_{2} \beta_{1}\right) a_{3}
$$

where $\alpha, \beta$ are the one-electron spin up and spin down functions, we may write, in a usual notation

$$
\mathbf{F}(3)=\sum_{\ell=0}^{\infty} \frac{\mathrm{f}_{\ell}(3)}{\mathbf{r}_{3}} \mathrm{P}_{\ell}\left(\mu_{3}\right)
$$

the position vector of electron 3 with respect to the target nucleus being $r_{3}=\left(r_{3}, \theta_{3}, \phi_{3}\right)$ and $\mu_{3}=\cos \theta_{3}$

The integrodifferential equation for the scattering functions $\mathfrak{f}_{\ell}\left(\mathbf{r}_{3}\right)$ is obtained by projecting out (1) on each partial wave in turn,

$$
\int \psi_{0}^{*}(1,2) \mathrm{P}_{\ell}^{*}\left(\mu_{3}\right) \mathrm{S}^{*}(123)[\mathrm{H}-\mathrm{E}] \Psi(123) \mathbf{d} \mathbf{r}_{1} \mathbf{d} \mathbf{r}_{2} \mathrm{~d} \hat{\mathbf{r}}_{3} \mathrm{~d} \mathrm{Spin}=0
$$

For simplicity we assume that the ground state function for the target is known exactly:

$$
\left(-\frac{1}{2} \nabla_{1}^{2}-\frac{1}{2} \nabla_{2}^{2}-\frac{z}{r_{1}}-\frac{z}{r_{2}}+\frac{1}{r_{12}}\right) \psi_{0}(12)=E_{z} \psi_{0}(12)
$$

and

$$
E=E_{z}+k^{2}
$$

We adopt a two-parameter (11) variational trial function

$$
\psi_{0}(1,2)=N\left(\mathrm{e}^{-\alpha \mathrm{r}_{1}-\beta \mathbf{r}_{2}}+\mathrm{e}^{-\beta \mathbf{r}_{1}-\alpha \mathrm{r}_{2}}\right)
$$


for the target. Then on carrying out the angular and spin integrations (6) yields

$$
\begin{aligned}
\left.\mathrm{L}_{\ell}^{0}\right) \mathrm{f}_{\ell}\left(\mathrm{r}_{3}\right)=\frac{16 \pi^{2} \mathrm{~N}^{2}}{(2 \ell+1)} & {\left[\delta \int_{0}^{\infty} \int_{0}^{\infty} \mathrm{f}_{0}(1) \mathrm{Z}_{00}(123) \mathrm{d} \mathrm{r}_{1} \mathrm{~d} \mathrm{r}_{2}\right.} \\
& \left.-2 \int_{0}^{\infty} \int_{0}^{\infty} \mathrm{f}_{\ell}(1) \mathrm{x}_{0 \ell}(123) \mathrm{dr} \mathrm{r}_{1} \mathrm{dr} \mathrm{r}_{2}\right]=\mathrm{R}_{\ell}\left(\mathrm{r}_{3}\right),
\end{aligned}
$$

where $L_{\ell}^{(0)}$ is the operator

$$
\mathrm{L}_{\ell}^{(0)}=\frac{\mathrm{d}^{2}}{\mathrm{dr^{2 }}}+\mathrm{k}^{2}-\frac{\ell(\ell+1)}{\mathrm{r}^{2}}-\mathrm{V}_{00}(3)
$$

The direct and exchange potentials are

$$
\begin{aligned}
& V_{00}(r)=-\frac{2 z}{r}+\frac{4 \pi^{2} N^{2}}{a^{3} \beta^{3} r}\left[\left[1-(1+\alpha r] e^{-a r}\right]+\left[1-(1+\beta r) e^{-\beta r}\right]\right. \\
& \left.+\frac{128 a^{3} \beta^{3}}{(\alpha+\beta)^{6}}\left[1-\left(1+\frac{1}{2} \overline{\alpha+\beta} \mathrm{r}\right) \mathrm{e}^{-(\alpha+\beta) \mathrm{r}}\right]\right] \\
& \mathrm{Z}_{00}=\frac{\mathrm{r}_{3}}{\mathrm{~N}} \psi_{0}^{*}(23) \mathrm{r}_{2}^{2}\left\{\left(\alpha^{2} \mathrm{r}_{1}-2 \alpha\right) \mathrm{e}^{-\alpha \mathrm{r}_{1}-\beta \mathrm{r}_{2}}+\left(\beta^{2} \mathrm{r}_{1}-2 \beta\right) \mathrm{e}^{-\beta \mathrm{r}_{1}-\alpha \mathrm{r}_{2}}\right. \\
& \left.+\frac{1}{\mathrm{~N}} \psi_{0}(1,2)\left[\mathrm{r}_{1} \mathrm{k}^{2}+2 z-\frac{2 \mathrm{r}_{1}}{\mathrm{r}_{12}^{7}}\right]\right\} \\
& x_{0} l=r_{1} r_{3} r_{2}^{2} \psi_{0}^{*}(1,2) \frac{2}{r_{13, \ell}} \psi_{0}(2,3), r_{13, l}=\left(\frac{r_{<}^{l}}{r_{>}^{l+1}}\right)_{13}
\end{aligned}
$$


No account has been taken of the polarizability of the target in this model, so we replace $L_{\ell}^{(0)}$ by

$$
L_{\ell}=L_{\ell}^{(0)}-2\left(v_{d}+v_{q}\right)
$$

where $v_{d}$ and $v_{q}$ are potentials behaving as $r^{-4}$ and $r^{-6}$ respectively at large r. They are chosen in the Bethe-Reeh form ${ }^{12}$

$$
\begin{aligned}
v_{d}(r)= & -\frac{9}{2 u^{4}}\left\{1-\frac{1}{3} e^{-2 u}\left[1+2 u+6 u^{2}+\frac{20}{3} u^{3}+\frac{4}{3} u^{4}\right]\right. \\
& \left.-\frac{2}{3} e^{-4 u}[1+u]^{4}\right\}, \\
v_{q}(r)= & -\frac{15}{2 y^{6}}\left\{1-2 y e^{-2 y}\left[1+2 y+\frac{4}{3} y^{2}+\frac{1}{3} y^{3}+\frac{2}{9} y^{4}\right.\right. \\
& \left.+\frac{1}{9} y^{6}+\frac{1}{18} y^{6}-\frac{1}{175} y^{7}+\frac{1}{135} y^{8}-\frac{2}{135} y^{9}\right] \\
& \left.+\frac{8}{135} y^{10} E i(-2 y)\right\} . \\
& +e^{-4 y}\left[1+4 y+\frac{20}{3} y^{2}+6 y^{3}+\frac{28}{9} y^{4}+\frac{8}{9} y^{5}+\frac{1}{9} y^{6}\right] \\
&
\end{aligned}
$$


where $\mathrm{E} i(-\mathrm{u})=-\mathrm{E}_{1}(\mathrm{u})$ and $\mathrm{E}_{1}(\mathrm{u})$ is the exponential integral, ${ }^{13}$

$$
u=z_{1} r, y=z_{2} r
$$

The parameters $z_{1}, z_{2}$ are chosen so that $\left(9 / z_{1}\right)^{4}=\alpha_{d},\left(15 / z_{2}\right)^{6}=a_{q}$, where $a_{d}, a_{q}$ are the dipole and quadrupole polarizabilities of the target respectively.

\section{Numerical Methods}

The integrodifferential equation

$$
\mathrm{L}_{\ell} \mathbf{f}_{\ell}(\mathrm{r})=\mathrm{R}_{\ell}(\mathbf{r})
$$

must be solved subject to the boundary conditions

$$
\mathrm{f}_{\ell}(0)=0, \mathrm{f}_{\ell}(\mathrm{r}) \underset{\mathrm{r} \rightarrow \infty}{\sim} \mathrm{k}^{-1 / 2} \sin \left(\mathrm{kr}+\frac{\mathrm{z}_{0}}{\mathrm{k}} \ln 2 \mathrm{kr}-\frac{1}{2} \ell \pi+\sigma_{\ell}+\delta_{\ell}\right)
$$

with

$$
\sigma_{\ell}=\arg 1^{7}\left(\ell+1-\frac{\mathrm{iz}_{0}}{\mathrm{R}}\right)
$$

and $z_{0}=(z-2)$. The quantity of interest is the non-couloumb part of the phase shift, $\delta_{\ell}$. We shall refer to it simply as the phase shift (for the $\ell^{\text {th }}$ partial wave).

The equation $\left(10^{\prime}\right)$ was solved by a non-iterative method. Writing

$$
\mathrm{f}_{\ell}(\mathrm{r})=\mathrm{P}(\mathrm{r})+\mu \mathrm{Q}(\mathrm{r})+\nu \mathrm{R}(\mathrm{r})
$$


where $P, Q$, R satisfy

$$
\begin{aligned}
& \mathrm{L}_{\ell} \mathrm{P}=\mathrm{F}(\mathrm{P}) \\
& \mathrm{L}_{\ell} \mathrm{Q}=\mathrm{F}(\mathrm{Q})+\mathrm{c}_{\ell} \mathrm{r}^{\ell+1} \mathrm{e}^{-a \mathrm{r}} \\
& \mathrm{L}_{\ell} \mathrm{R}=\mathrm{F}(\mathrm{R})+\mathrm{c}_{\ell} \mathrm{r}^{\ell+1} \mathrm{e}^{-\beta \mathrm{r}}, \mathrm{c}_{\ell}=\frac{16 \pi^{2} \mathrm{~N}^{2}}{(2 \ell+1)}
\end{aligned}
$$

and $\mathrm{F}(\phi)$ involves linear combinations of integrals over the range $(0, \mathrm{r})$ only, the parameters $\mu, \nu$ may be determined in terms of certain infinite integrals once $P(r), Q(r)$ and $R(r)$ are known. For He this procedure yields phase shifts identical to those obtained previously ${ }^{2}$ by an iterative method. The ordinary differential equations (18) were solved by a Fox-Goodwin predictor-corrector method.

The solutions were normalized by the Strömgren procedure ${ }^{14}$ as given by Burgess. His procedure for determining the phase shift when a polarization potential is present may be extended easily to the case of a negative ion $\left(z_{0}=-1\right)$. At large $r$,

$$
f_{\ell}(r) \underset{r \rightarrow \infty}{\sim} k^{-1 / 2} \sin \left[\phi(r)+\delta_{\ell}\right]
$$

where to sufficient accuracy

$$
\phi(r)=\phi_{1}(r)+\frac{1}{2} \Phi(r) .
$$


Defining

$$
\rho=\left|z_{0} \mathbf{r}\right|, \beta=\left|z_{0} k\right|
$$

we have (the \pm sign being that of $z_{0}$ ),

$$
\begin{gathered}
\phi_{1}^{ \pm}=\chi(\rho)^{ \pm} \pm \frac{1}{\beta} \ln \left[\left(\beta^{2} \rho+\beta \chi^{ \pm} \pm 1\right) / \beta\right]-\frac{1}{2} \rho \pi+\sigma \ell \mp \frac{1}{\beta} \\
\pm \frac{5(\rho \mp c)}{24 \chi^{( \pm) 3}}-\frac{\chi^{ \pm}\left(3 \beta^{2}+4\right)+\beta \rho\left(3 \beta^{2} c+2\right) \pm \beta c}{24 \chi\left(1+\beta^{2} \mathrm{c}\right)\left(\chi^{ \pm}+\beta \rho\right)} \\
+\frac{\left(\mathrm{c}+\frac{1}{8}\right)}{\sqrt{c}} \cos ^{-1} \mathrm{~T}^{ \pm}(\rho),
\end{gathered}
$$

with

$$
X^{ \pm}=+\left(\beta^{2} \rho^{2} \pm 2 \rho-c\right)^{1 / 2}, T^{ \pm}(\rho)=\frac{\beta c X^{ \pm} \mp c+\rho}{\rho\left(1+\beta^{2} c\right)}, c=\ell(l+1)
$$

The polarization term is

$$
\begin{aligned}
\Phi(\rho) & =\frac{z_{0}^{2} a_{d}}{4 c^{2}}\left[\frac{3(2 \rho \mp c)}{\rho\left(\chi^{ \pm}+\beta \rho\right)}+\frac{c \chi^{ \pm}}{\rho^{2}}-\frac{\left(3+\beta^{2} c\right)}{\sqrt{c}} \cos ^{-1} \mathrm{~T}^{ \pm}(\rho)\right], c \neq 0 \\
& =-\frac{2 z_{0}^{2} \alpha_{d}\left(9 \beta \rho \chi^{ \pm}+11 \beta^{2} \rho^{2} \pm 6 \rho\right)}{15 \rho^{2}\left(\chi^{ \pm}+\beta \rho\right)^{3}}, c=0 .
\end{aligned}
$$

When $\mathbf{c}=0$ the $\cos ^{-1}$ term in (20) is replaced by $0.25\left(\chi^{ \pm}+\beta \rho\right)^{-1}$. No special treatment of the quadrupole potential is required. Dipole and quadrupole polarizabilities for $\mathrm{H}^{-}$are given by Stewart. ${ }^{5}$ The value of the quadrupole polarizability used is uncertain by $\pm 20 \%$, but in view of its small effect, this is not 
significant. The dipole polarizability used for $\mathrm{Li}^{+}$is the Hartree-Fock value of Lahiri and Mukkerji ${ }^{16}$ and should be accurate; its quadrupole polarizability was taken as zero. The calculated phase shifts for $\mathrm{H}^{-}$are shown in Table 2. The program actually calculates $\tan \delta_{\ell}$, and $\delta_{\ell}(\bmod \pi)$. The calculations indicated that if the phase shift was defined to go to zero in the high energy limit, then the $\mathrm{s}$-wave phase shift must be chosen to be $\pi$ in the zero-energy limit. This was confirmed by comparing the numerical solution for $\mathrm{k}^{2}=0.05, l=0$ with the corresponding pure couloumb solution. An extra node is present in the calculated solution at small $\mathrm{r}^{(21)}$. Physically this occurs because the incoming electron cannot enter the filled $1 \mathrm{~s}-\mathrm{shell}$, and there is in our model, no bound (1s) $2 \mathrm{~s}$ state of $\mathrm{H}^{--}$. A similar situation occurs for electron scattering by He, but not for $\mathrm{Li}^{+}$. In the latter case the $(1 \mathrm{~s})^{2} 2 \mathrm{~s}$ state is the ground configuration of $\mathrm{Li}$, and the zero energy s-wave phase shift is found to be 1.261 (see below). The s-wave phase shifts for all these systems are shown in Fig. 1. In Fig. 2 we show $\tan \delta_{0}$ for $\mathrm{H}^{-}$both including $(\mathrm{d}+\mathrm{q})$ and excluding (d) the quadrupole potential. The local potentials occurring in Eq. $\left(10^{\prime}\right)$ are shown in Fig. 3. They are strongly repulsive at large $r$ but the effect of the attractive polarization potentials is to produce a broad positive maximum in $V(r)$ from $r=2.0$ to $r=10.0$ with a maximum value of $0.2 \mathrm{Ry}$. The potential $\mathrm{V}(\mathrm{r})$ then becomes strongly attractive for $\mathrm{r} \leq 2.0 \mathrm{a}_{0}$. There appears to be a possibility of trapping for low energy electrons. We therefore computed $\tan \delta_{0}$ for $0.05 \leq \mathrm{k}^{2} \leq 0.2$ Ry at intervals of 0.01 in $\mathrm{k}^{2}$ (i.e. $0.136 \mathrm{eV}$ ). No evidence for an $\mathrm{s}$-wave resonance 
was observed, but in any event no resonance appreciably narrower than $0.1 \mathrm{eV}$ would have been found. Herzenberg and Lau ${ }^{17}$ have used our model (with a different form of polarization potential) to investigate s-wave scattering of slow electrons in $\mathrm{He}$, and obtain a resonance by suitably modifying the strength of the exchange terms. Peterkop ${ }^{18}$ has also reported an s-wave resonance in He, in a one channel model.

Although the calculated results for $\mathrm{He}$ and $\mathrm{Li}^{+}$are in excellent agreement with other calculations (and for He, with experiment), it might appear that the model would be less satisfactory for $\mathrm{H}^{-}$, with its extremely large polarizability. In fact the calculated phase shifts in $\mathrm{He}$ and $\mathrm{Li}^{+}$are also dominated by the polarizability potential (for small $\ell$ and $\mathrm{k}^{2}$ ) and are substantially in error when polarization is neglected. We would therefore expect our $\mathrm{H}^{-}$results to be of comparable accuracy.

The effect of introducing a quadrupole polarization is to slightly increase all the phase shifts, in general by less than $10 \%$. The highest value of $\mathrm{k}^{2}$ at which the p-wave phase shift passes through $\pi / 2$ increases from 2.0 to 2.45 when $\alpha_{q}$ changes from zero to 1300 . For given $k^{2}$, the phase shifts decrease slowly with $\ell(\ell \geq 1)$.

Calculated phase shifts for $\mathrm{Li}^{+}$are displayed in Table 3. When the polarization potentials are omitted the calculated values are in very close agreement 
with these obtained in a Hartree-Fock calculation. ${ }^{10}$ This confirms the result obtained for $\mathrm{He}^{1,2}$ that provided the target ground state wave function used gives a binding energy at least as good as the Hartree-Fock value, its detailed behavior is not important in determining the low energy phase shifts. In particular it appears that short range $l=0$ correlation in the target is not important. The $\mathrm{Li}^{+}$ $\mathrm{p}$-wave phase shift obtained when polarization is included tends to a threshold value $(0.170)$ in close agreement with that $(0.188)$ obtained using the quantum defect method, ${ }^{19}$ but rather larger than the no-polarization value of 0.110 .

The zero energy s-wave phase shift is 1.261 where the quantum defect method gives $1.232 \pm 0.005$. The d-wave phase shift at threshold, is like its quantum defect value, very small (approximately $3.8 \times 10^{-3}$ ).

We have computed angular distributions of elastically scattered electrons for $\mathrm{H}^{-}$, where deviations from pure Couloumb scattering would be expected to be significant.

The non-couloumb contribution $f_{N}(\theta)$ to the scattering amplitude $f(\theta)$ may be written

$$
\mathrm{f}_{\mathrm{N}}(\theta)=-\frac{\mathrm{i}}{2 \mathrm{k}} \sum_{\ell=0}^{\infty}(2 \ell+1) \mathrm{e}^{2 \mathrm{i} \sigma \ell}\left(\mathrm{e}^{2 \mathrm{i} \delta \ell}-1\right) \mathrm{P}_{\ell}(\cos \theta)
$$

and because of the large polarizability of $\mathrm{H}^{-}$a comparatively large number of terms must be retained in the summation over $\ell$ in (22). We find that for 
$\mathrm{k}^{2} \leq 3.0$, the sum has converged to better than $1 \%$ if it is cut off at $\ell=30$. For $\ell \geq 4$ the exchange terms in $\left(10^{\prime}\right)$ may be ignored; phase shifts are then obtained from the suitably normalized solutions of

$$
L_{\ell}(r) f_{\ell}(r)=0
$$

In table (2b) we give phase shifts in this approximation for $\ell=4$ (1) 10 at $\mathrm{k}^{2}=0.5,0.75,1.0,3.0$

Rather than displaying the scattering intensity $I(\theta)=|\mathbf{f}(\theta)|^{2}$ it is convenient first to show $R(\theta)$, the ratio of $I(\theta)$ to the pure coloumb intensity $I_{c}(\theta)$. Writing $\alpha=1 / \mathrm{k}$ we have $\mathrm{e}^{20}$

$$
\mathbf{R}\left(\theta, \mathrm{k}^{2}\right)=|1+\mathrm{N}|^{2}
$$

with (for $\mathrm{H}^{-}$),

$\mathrm{N}=-2 \mathrm{k} \sin ^{2} \frac{\theta}{2}\left\{\exp \mathrm{i} \alpha \ln \left(\sin ^{2} \frac{\theta}{2}\right)\right\} \sum_{\ell=0}^{\ell}(2 \ell+1) \mathrm{e}^{2 \mathrm{i} \delta \ell} \sin \delta_{\ell} \mathrm{e}^{2 \mathrm{i}\left(\sigma \ell-\sigma_{0}\right)} \mathrm{P}_{\ell}(\cos \theta)$

The calculated values of $\mathrm{R}\left(\theta, \mathrm{k}^{2}\right)$ for $\mathrm{k}^{2}=0.50,0.75,1.0,3.0$ are shown in Fig. 4.

The most significant features are the sharp dip near $\theta=0.4$, the oscillations in the range $1.0 \leq \theta \leq 2.5$ and the strong enhancement in the backward direction for $k^{2}=0.5$. The fine detail of the calculated oscillations in $R\left(\theta, k^{2}\right)$ at intermediate angles may not be significant. 
In Figs. 5a, 5b we show $I(\theta)$ and $I_{c}(\theta)$ for $R^{2}=0.5$ and 1.0 over the angular range $0.3 \leq \theta \leq \pi$. The calculated intensity is a factor of five lower than the couloumb intensity at $40^{\circ}$, but for $\mathrm{k}^{2}=0.5$ it is a factor of four higher in the backward direction.

\section{Acknowledgments}

Thanks are due to Dr. A. Temkin for several helpful discussions. 


\section{References}

1. R. W. LaBahn and J. Callaway, Phys. Rev., 135, A 1539, (1964), 147, 28 (1966).

2. J. H. Williamson and M. R. C. McDowell, Proc. Phys. Soc. (London), $\underline{85}$, 719 (1964).

3. J. Lawson, H. S. W. Massey, J. Wallace, and D. Wilkinson, Proc. Roy. Soc. (London) A $\underline{294}, 149$ (1966).

4. R. T. Pu and E. S. Chang, Phys. Rev., 151, 31 (1966).

5. D. E. Golden and H. W. Bandel, Phys. Rev., 138, A14 (1965).

6. L. S. Frost and A. V. Phelps, Phys. Rev., 136, A 1538 (1964).

7. G. Tisone and L. M. Branscomb, Phys. Rev. Letters, 17, 236 (1966).

8. D. F. Dance, M. F. A. Harrison, and R. D. Rundel, Proc. Roy. Soc., (London), A 299, 525 (1967).

9. J. H. Williamson and M. R. C. McDowell, Phys. Letters, $\underline{4}, 159$ (1963).

10. E. S. Chang, private communication.

11. R. D. Hurst, J. D. Gray, G. H. Briguran, and F. A. Matsen, Mol. Phys., 1, 189 (1958). 
12. H. Reeh, Zeit Naturforsch, $\underline{15} \underline{\mathrm{A}}, 377$ (1960).

13. M. Abramowitz and I. A. Stegan, Handbook of Mathematical Functions (Dover, N. Y.), (1965).

14. A. Burgess, Proc. Phys. Soc. (London, 81, 442 (1963).

15. A. L. Stewart, Adv, in Phys., $\underline{12}, 47$ (1963).

16. J. Lahiri and A. Mukherji, J. Phys. Soc. (Japan), 21, 1128 (1966).

17. H. S. N. Lau and A. Herzenberg, Abstracts of 5th International Conference on the Physics of Electronic and Atomic Collisons, Leningrad, p. 261 (Nauk, Leningrad), 1967.

18. R. Peterkop, Abstracts of 5th International Conference on the Physics of Electronic and Atomic Collisons, Leningrad, p. 391 (Nauk, Leningrad), 1967.

19. M. J. Seaton, Mon. Notes Roy. Astronom. Soc., $\underline{118}, 508,1958$.

20. N. F. Mott and H. S. W. Massey, Theory of Atomic Collisons (3rd Ed.), (O.U.P.) (1965).

21. P. Swan, Proc. Roy. Soc. (London) A 228,10 (1955), private communication (1958).

22. A. Temkin, J. Math. Phys., $\underline{2}, 336$ (1961). 


\section{Figure Captions}

Fig. 1 Calculated s-wave phase shift for elastic scattering of slow electrons by the two electron systems indicated.

Fig. 2 Tan $\delta_{0}\left(\mathrm{k}^{2}\right)$ for elastic scattering by $\mathrm{H}^{-}$. Full curve $\alpha_{\mathbf{q}}=\mathbf{0}$, dashed curve $a_{q} \neq 0$.

Fig. 3 The local potentials occurring in Eq. $\left(10^{\prime}\right)$ for $\mathrm{Z}=1\left(\mathrm{H}^{-}\right)$.

Fig. 4 Calculated values of $\mathbf{R}\left(\theta, \mathbf{k}^{2}\right)$, the ratio of $\mathbf{I}(\theta)$ to the Couloumb intensity $I_{c}(\theta)$ for $H^{-}$, (1) $k^{2}=0.5$ (2) $k^{2}=0.75$ (3) $k^{2}=1.0$ (4) $\mathrm{k}^{2}=3.0$.

Fig. 5 Calculated differential cross sections for scattering by $\mathrm{H}^{-}$. The full curve is $I_{c}(\theta)$, the dashed curve $I(\theta)$. (a) $k^{2}=0.5$, (b) $k^{2}=1.0$. 


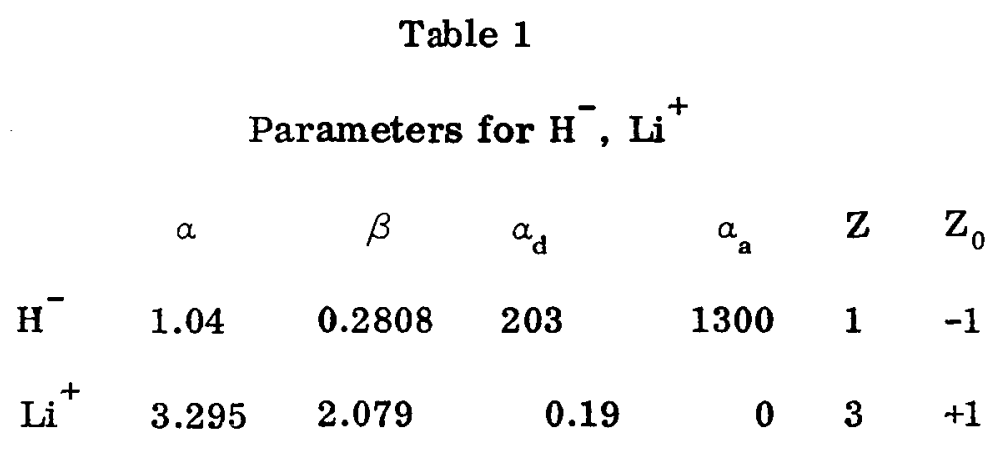


Table 2a

S-Wave Phase Shifts for $\mathrm{H}^{-}$

$\tan \eta_{0}$

$\eta_{0}$

$\mathrm{k}^{2}$

d

$d+q$

$d+q$

0.05

$8.19,-3$

$8.22,-3$

3.150

0.06

$1.26,-2$

$1.27,-2$

3.154

0.07

$1.84,-2$

$1.85,-2$

3.160

0.08

$2.51,-2$

$2.54,-2$

3.167

0.09

$3.26,-2$

$3.31,-2$

3.175

0.10

$4.06,-2$

$4.13,-2$

3.183

0.25

$2.22,-1$

$2.50,-1$

3.389

0.50

$4.71,-1$

$5.59,-1$

3.650

0.75

$3.49,-1$

$4.20,-1$

3.540

1.0

$1.74,-1$

$2.30,-1$

3.369

2.0

$-3.49,-1$

$-3.07,-1$

2.844

3.0

$-7.43,-1$

$-6.97,-1$

2.510

(d indicates dipole potential only $\mathrm{d}+\mathrm{q}$ indicates dipole plus quadrupole potentials) 
Table 2b

Calculated $\mathrm{H}^{-}$Phase Shifts, Notation as in Table 2a

$$
\ell=1 \quad l=2
$$

$\mathrm{k}^{2}$

d

$d+q$

d

$$
d+q
$$

0.10

$4.32,-2$

$4.38,-2$

$2.95,-2$

$2.99,-2$

0.55

$1.49,-1$

1.53.-1

$7.41,-2$

$8.08,-2$

0.20

$3.72,-1$

$4.00,-1$

$1.59,-1$

$1.63,-1$

0.25

$7.28,-1$

$8.12,-1$

$2.64,-1$

$2.76,-1$

0.50

1.71

1.82

$8.13,-1$

$8.78,-1$

0.75

1.78

1.86

1.04

1.11

1.0

1.75

1.82

1.11

1.18

1.5

1.65

1.70

1.12

1.17

2.0

1.57

1.61

1.09

1.14

3.0

1.44

1.46

1.03

1.07 
Table 2c

Dipole Plus Quadrupole Potentials Only

\begin{tabular}{|c|c|c|c|}
\hline $\mathrm{k}^{2}$ & $l=3$ & $\ell=4$ & $\begin{array}{c}\ell=4 \\
\text { no exchange }\end{array}$ \\
\hline 0.25 & 0.139 & 0.0798 & - \\
\hline 0.5 & 0.400 & 0.221 & - \\
\hline 0.75 & 0.667 & 0.403 & 0.390 \\
\hline 1.0 & 0.768 & 0.503 & 0.497 \\
\hline 1.5 & 0.858 & 0.624 & - \\
\hline 2.0 & 0.869 & 0.669 & - \\
\hline 3.0 & 0.846 & 0.687 & - \\
\hline
\end{tabular}


Table 2d

Phase Shifts for $\mathrm{H}^{-}, \ell \geq 4$, no exchange

(dipole plus quadrupole potential only)

\begin{tabular}{cccccccc}
$\mathrm{k}_{0}^{2} \ell$ & 4 & 5 & 6 & 7 & 8 & 9 & 10 \\
\cline { 3 - 8 } 0.50 & 0.230 & 0.147 & 0.0944 & 0.0637 & 0.0449 & 0.0327 & 0.0244 \\
0.75 & 0.390 & 0.244 & 0.158 & 0.107 & 0.075 & 0.0540 & 0.0403 \\
1.0 & 0.497 & 0.329 & 0.221 & 0.152 & 0.107 & 0.0775 & 0.0577 \\
3.0 & 0.687 & 0.552 & 0.447 & 0.360 & 0.288 & 0.231 & 0.185
\end{tabular}


Table 3

Phase Shifts for $\mathrm{Li}^{+}$, Dipole Potential Only

$\begin{array}{lccccc}\mathrm{k}^{2} & \ell=0 & \ell=1 & \ell=1, \mathrm{H} . \mathrm{F} . \quad l=1, a_{\mathrm{d}}=0 & \ell=2 \\ 0 & (1.261) & (0.170) & (0.110) & - & (0.0038) \\ 0.04 & 1.258 & 0.173 & 0.113 & - & 0.0044 \\ 0.09 & 1.255 & 0.177 & 0.117 & - & 0.0052 \\ 0.16 & 1.248 & 0.182 & 0.120 & 0.122 & 0.0063 \\ 0.25 & 1.241 & 0.189 & 0.126 & - & 0.0074 \\ 0.36 & 1.230 & 0.195 & 0.132 & 0.133 & 0.0088 \\ 0.49 & 1.221 & 0.202 & 0.140 & 0.140 & 0.0108 \\ 0.64 & 1.209 & 0.210 & 0.147 & 0.146 & 0.0135 \\ 0.81 & 1.194 & 0.217 & 0.153 & - & 0.0157 \\ 1.0 & 1.178 & 0.224 & 0.161 & - & 0.0182\end{array}$

(1) Values for $\mathrm{k}^{2}=0$ are obtained by graphical extrapolation.

(2) For the p-wave phase shift the successive columns show the results (i) in our model with $\alpha_{d}=0.19$, (ii) in a HartreeFock calculation, ${ }^{10}$ (iii) in our model with $\alpha_{d}=0$. 


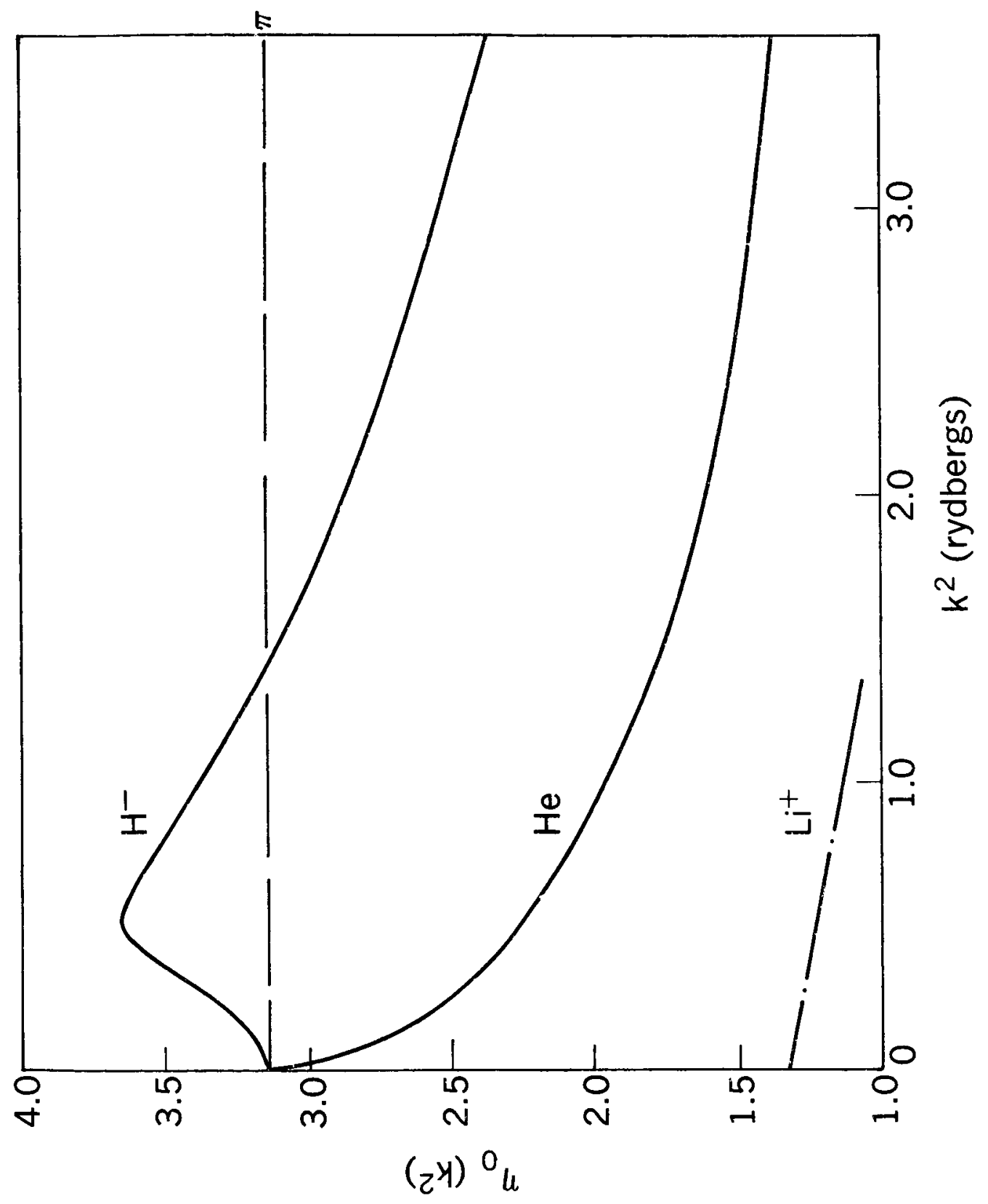

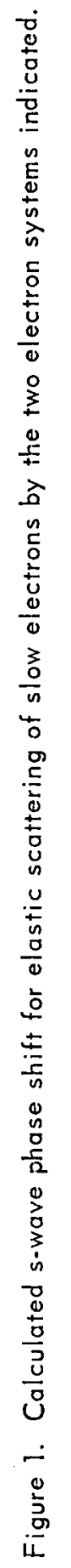




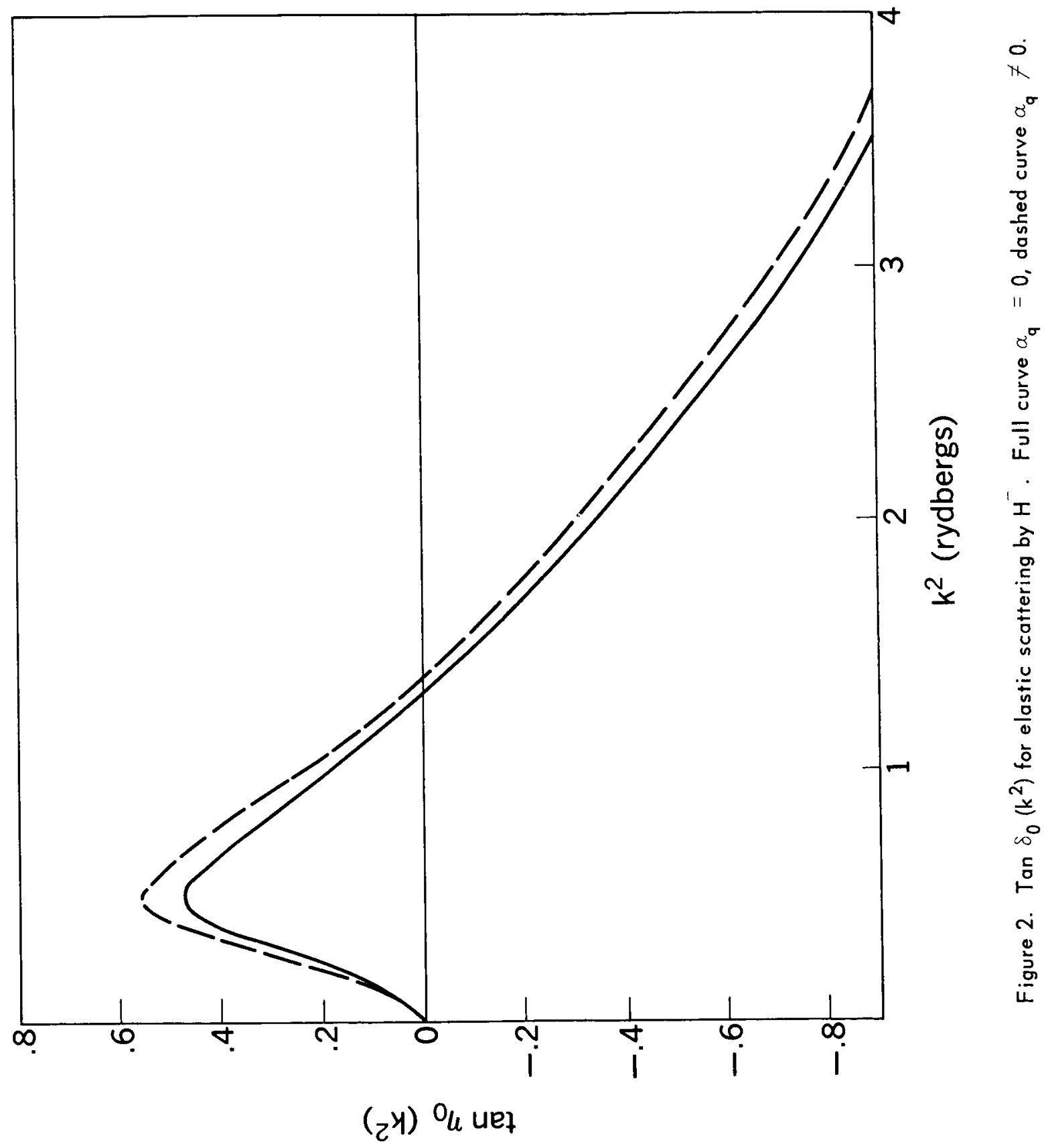




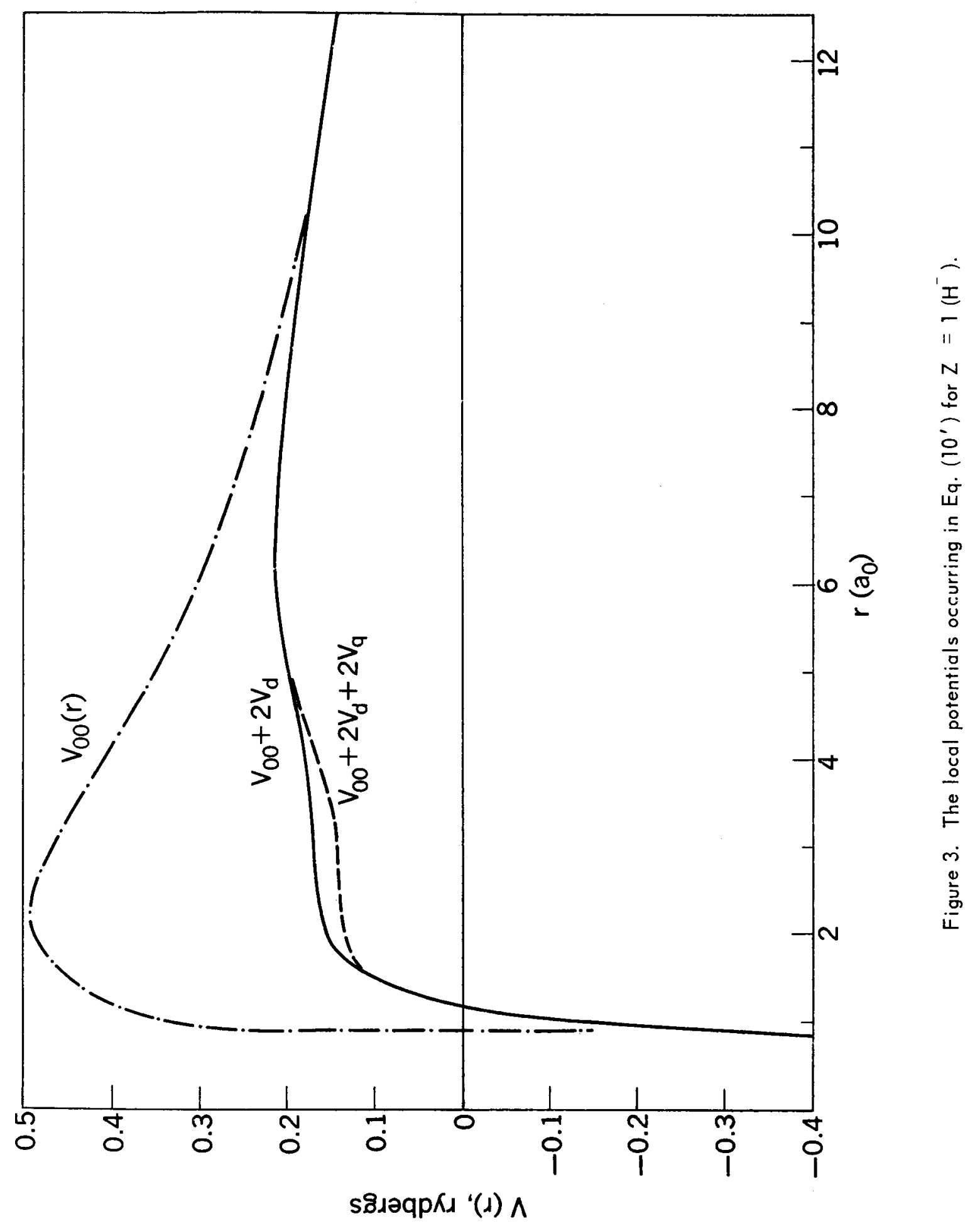




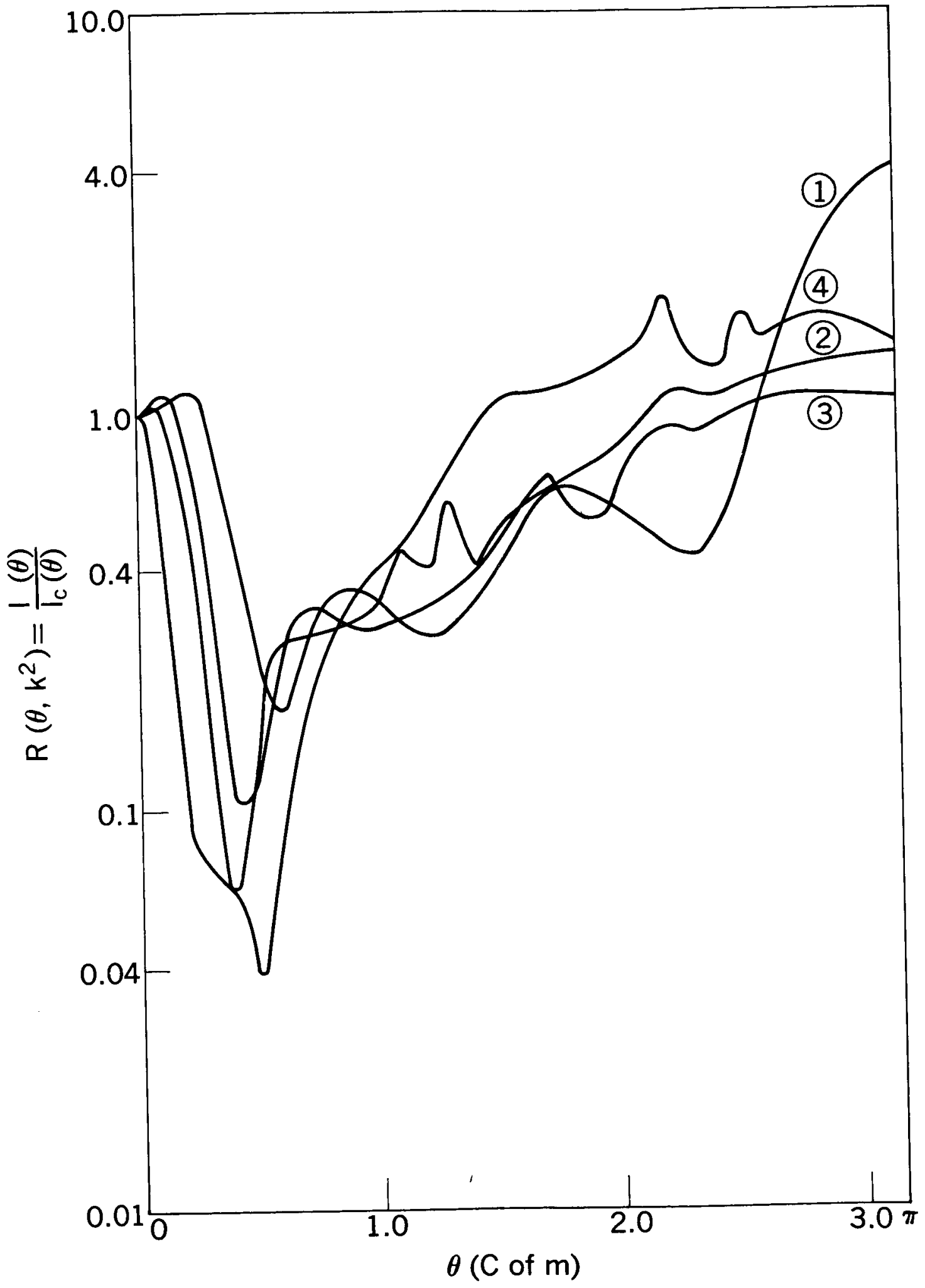

Figure 4. Calculated values of $R\left(\theta, \mathrm{k}^{2}\right)$, the ratio of $\mathrm{I}(\theta)$ to the Couloumb intensity $i_{c}(\theta)$ for $H^{-},(1) k^{2}=0.5(2) k^{2}=0.75(3) k^{2}=1.0(4) k^{2}=3.0$. 


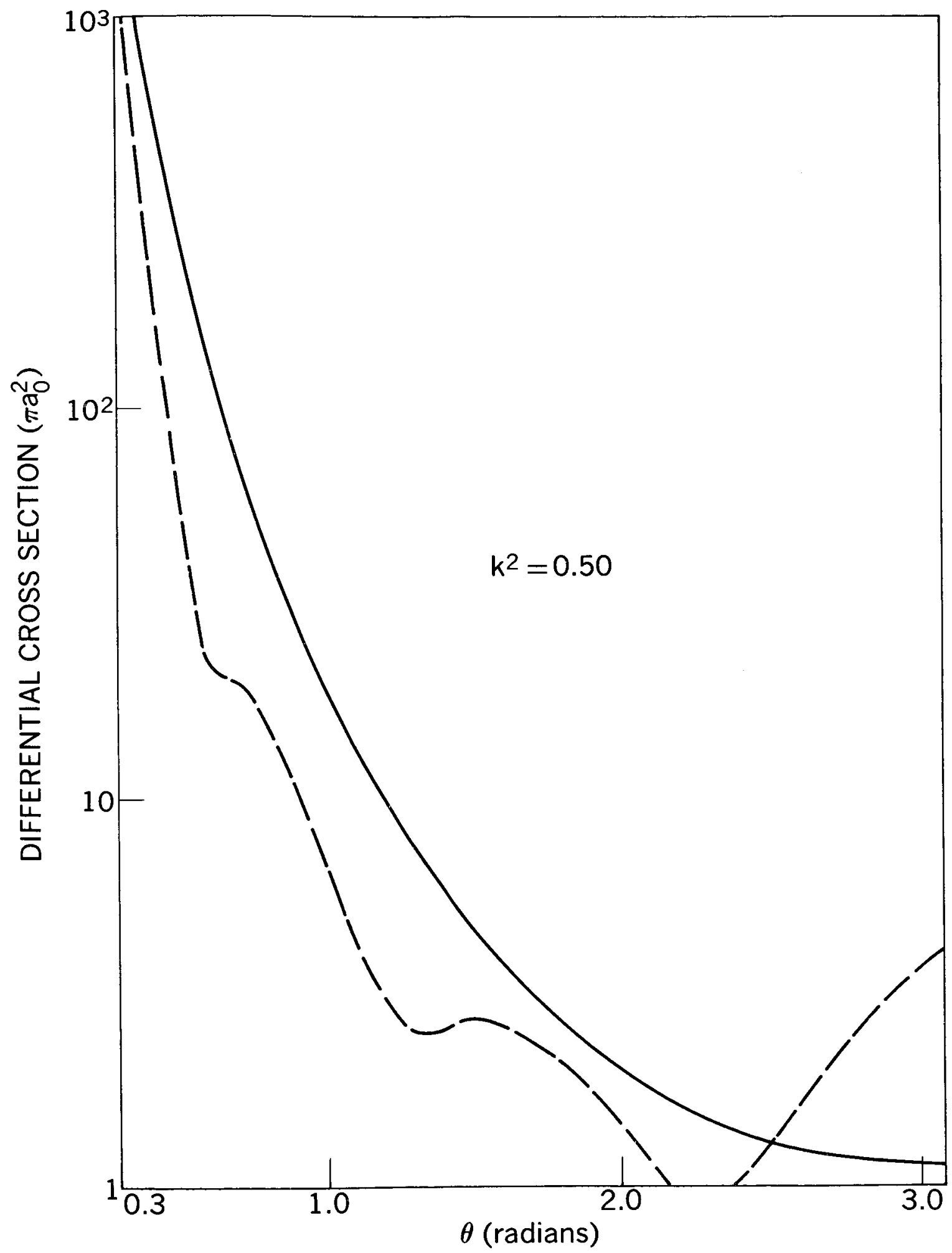

Figure 5a. Calculated differential cross sections for scattering by $\mathrm{H}^{-}$. The full curve is $I_{c}(\theta)$, the dashed curve $I(\theta)$. (a) $\left.\mathrm{k}^{2}=0.5, \mathrm{~Kb}\right) \mathrm{k}^{2}=1.0$. 


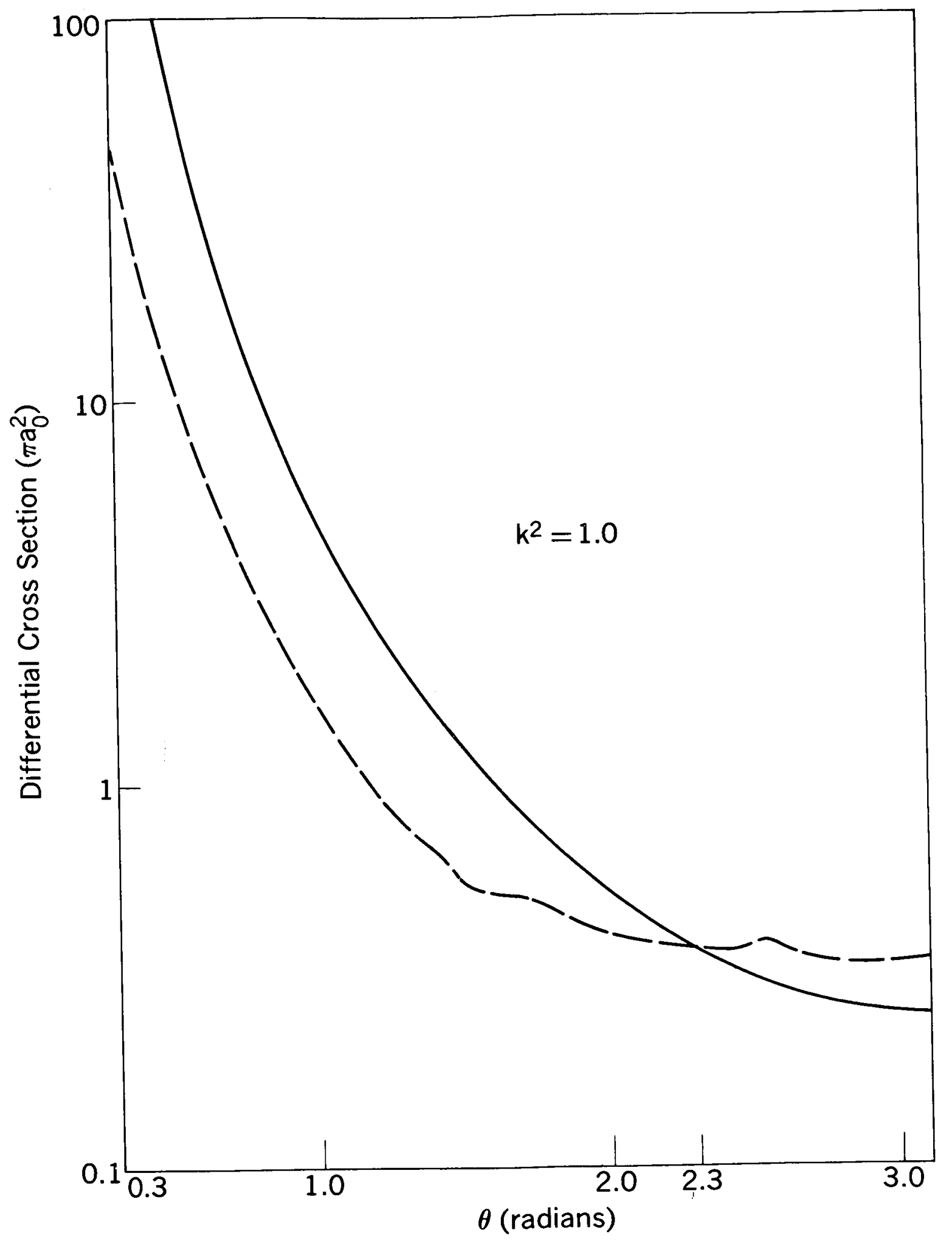

Figure 5b. Calculated differential cross sections for scattering by $\mathrm{H}^{-}$. The full curve is $\mathrm{I}_{c}(\theta)$, the dashed curve $\mathrm{I}(\theta)$. (a) $\mathrm{k}^{2}=0.5,(\mathrm{~b}) \mathrm{k}^{2}=1.0$. 